\title{
RAMAN SPECTROSCOPY OF HUMAN HEMOGLOBIN FOR DIABETES DETECTION
}

\author{
JUQIANG LIN*,†, JINYONG LIN**, ZUFANG HUANG*, \\ PENG LU*, JING WANG*, XUCHAO WANG* and RONG CHEN* \\ *Institute of Laser and Optoelectronics Technology \\ Fujian Provincial Key Laboratory for Photonics Technology \\ Key Laboratory of Optoelectronic Science and \\ Technology for Medicine of Ministry of Education \\ Fujian Normal University, Fuzhou 35000\%, P. R. China \\ †jqlin@fjnu.edu.cn
}

Received 24 May 2013

Accepted 11 September 2013

Published 18 October 2013

\begin{abstract}
Glycated hemoglobin (HbA1c) has been increasingly accepted as the gold standard for diabetes monitoring. In this study, Raman spectroscopy was tentatively employed for human hemoglobin $(\mathrm{Hb})$ biochemical analysis aimed at developing a simple blood test for diabetes monitoring. Raman spectroscopy measurements were performed on hemoglobin samples of patients $(n=39)$ with confirmed diabetes and healthy volunteers $(n=37)$. The tentative assignments of the measured Raman bands were performed to compare the difference between these two groups. Meanwhile, principal component analysis (PCA) combined with linear discriminant analysis (LDA) were employed to develop effective diagnostic algorithms for classification between normal controls and patients with diabetes. As a result, the spectral features of these two groups demonstrated two distinct clusters with a sensitivity and specificity of $92.3 \%$ and $73 \%$, respectively. Then the effectiveness of the diagnostic algorithm based on PCA-LDA technique was confirmed by receiver operating characteristic (ROC) curve. The area under the ROC curve was 0.92 , indicating a good diagnostic result. In summary, our preliminary results demonstrate that proposing Raman spectroscopy can provide a significant potential for the noninvasive detection of diabetes.
\end{abstract}

Keywords: Glycated hemoglobin; Raman spectroscopy; PCA-LDA.

\section{Introduction}

Diabetes, derived from the defect that human body fails to produce or to respond to insulin which regulates glucose fluctuation, is a chronic disease. It is characterized by blood glucose increase. With the deterioration of this disease, it will cause many

$\dagger$ Corresponding author.

*These co-first authors contributed equally to the work.

This is an Open Access article published by World Scientific Publishing Company. It is distributed under the terms of the Creative Commons Attribution 3.0 (CC-BY) License. Further distribution of this work is permitted, provided the original work is properly cited. 
diabetic complications, such as diabetic nephropathy, diabetic retinopathy, atherosclerosis and other perivascular diseases. ${ }^{1,2}$ It is well known that all kinds of diabetes are incurable. Therefore, proper monitoring of glycemic control becomes the most important thing. Recently glycated hemoglobin (HbA1c) as the new standard for diabetes monitoring has become a research hot spot. In diabetic blood, high concentration of blood glucose resulted in the formation of glycohemoglobin which mainly stemmed from the nonenzymatic glycation of the Nterminal valine on the $\beta$ chain of $\mathrm{Hb}^{3}$ Meanwhile, the lifecycle of red blood cell (RBC) is about 120 days, which determines that $\mathrm{HbA1c}$ can reflect the average blood glucose level over 2-3 months. So HbA1c can be used to monitor long-term glucose control in diabetes, making it possible to realize early diagnosis of diabetes.

Recently, Raman spectroscopy (RS) was widely used as a powerful diagnostic tool in biomedical research field for its characteristic of providing fingerprint information of the molecular composition and structure of a sample, such as proteins, lipids and so on. ${ }^{4}$ For diabetes diagnosis, Han et al. ${ }^{5}$ used RS to analyze the serum of diabetic patients and Barman et $a l .{ }^{6}$ used it to detect the glycated hemoglobin in hemolysate model formed by twocomponent mixture ( $\mathrm{Hb}$ and $\mathrm{HbA1c}$ ). To the best of our knowledge, there is no report on using $\mathrm{RS}$ of human hemoglobin - a multicomponent mixture - for diabetes detection.

In this paper, we applied RS to analyze the biochemical component of human hemoglobin for diabetes detection. Principal component analysis (PCA) and linear discriminant analysis (LDA) were employed to develop effective diagnostic algorithms for classification between normal controls and patients with diabetes.

\section{Materials and Methods}

\subsection{Patients and preparation of human $\mathrm{Hb}$ samples}

The whole blood samples used for preparing $\mathrm{Hb}$ were collected from 76 individuals, consisting of 39 patients with confirmed clinical diagnosis of type II diabetes mellitus (HbA1c $\geqq 6.5 \%)$ and 37 healthy volunteers. The whole blood samples of diabetes were provided by First Hospital of Fuzhou. Ethical approval was obtained for studying the human $\mathrm{Hb}$.
The condition of drawing blood is as follows: A single 3-Ml fasting venous blood was drawn to a EDTA anticoagulation tube between 7:00 and 8:00 in the morning. Blood plasma and white cells were carefully removed with a pipettor to obtain the RBCs by centrifugation at $3000 \mathrm{rpm}, 4^{\circ} \mathrm{C}$ for $10 \mathrm{~min}$. The volume of RBCs used in our study was $300 \mu$ l. To purify the RBCs, phosphate buffered saline (PBS) was added to wash the RBCs for at least three times. Then the residual PBS was removed by the same condition of centrifugation $\left(3000 \mathrm{rpm}\right.$, at $4{ }^{\circ} \mathrm{C}$ for $10 \mathrm{~min}$ ). After purification, deionized water which was pre-cooled at $4^{\circ} \mathrm{C}$ was added into RBCs in a volume ratio of $3: 1$ (water/packed RBCs) to make cells rupture by uptaking excessive water. RBCs were lysed completely after about $30 \mathrm{~min}$ of storage at $4^{\circ} \mathrm{C}$ in refrigerator. Then, the diluted $\mathrm{Hb}$ solution was centrifuged at 15,000 rpm for $10 \mathrm{~min}$ to remove RBCs membrane. $\mathrm{Hb}$ Samples were frozen at $-80^{\circ} \mathrm{C}$ before Raman measurement.

After the preparation of $76 \mathrm{Hb}$ samples, a 5- $\mu \mathrm{l}$ drop of $\mathrm{Hb}$ sample was transferred onto a rectangle aluminum plate and left in the air for drying naturally before Raman analysis. Figure 1 illustrates the schematic of $\mathrm{Hb}$ sample preparation for Raman analysis.

\section{2. $R S$ measurements}

A confocal Raman micro-spectrometer (Renishaw, Great Britain) with a $785 \mathrm{~nm}$ diode laser excitation was employed for the collection of Raman spectra. In our experiment, the range of Raman spectra was set from 400 to $1800 \mathrm{~cm}^{-1}$ and the Raman signals were collected from the edge of the $\mathrm{Hb}$ drop coating ring [shown in Fig. 1(c)] with an integration time of $10 \mathrm{~s}$ and accumulated four times. Before measurements, the wavelength position and instrumental response were calibrated by the $520 \mathrm{~cm}^{-1}$ vibration band of a silicon wafer.

\subsection{Data analysis}

The original data of Raman spectra were first treated using the program previously devised by Zhao et al. ${ }^{7}$ to remove the fluorescence background noise. Then, each of the spectra was normalized to the integrated area under the curve from 400 to $1800 \mathrm{~cm}^{-1}$. After these pretreatment, the ultimate spectrum was able to better analyze spectra shape for the reason that it eliminated the difference of 


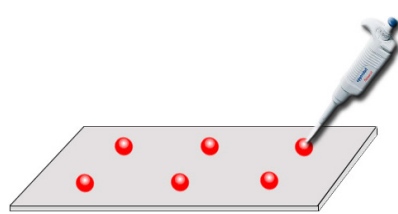

(a)

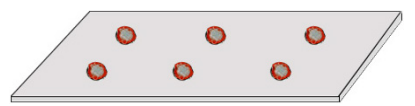

(b)

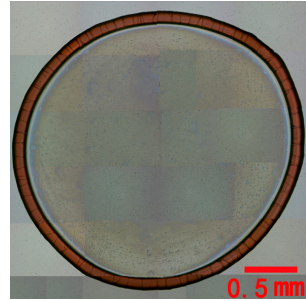

(c)

Fig. 1. Schematic of hemoglobin samples preparation for Raman measurement. (a) A 5- $\mu$ l drop of Hb sample was transferred onto a rectangle aluminum plate, respectively, then (b) the drop was left in the air for drying naturally. (c) Composite photograph showing the drop coating ring pattern produced by air-drying $\mathrm{Hb}$ sample solution on a rectangle aluminum slide.

absolute spectral intensity. ${ }^{8}$ At last, to extract and compare the spectral shape differences between diabetes group and health group, the multivariate statistic method PCA and LDA was employed.

PCA models a variance-covariance structure of a data matrix which is able to extract the eigenvalues - principal component (PC). ${ }^{9}$ Each $\mathrm{PC}$ is a linear combination of the $n$-independent wavenumber variables $\chi_{1} \ldots \chi_{2}$. For example:

$$
P C 1=a_{1} \chi_{1}+\cdots+a_{n} \chi_{n} .
$$

It is worth noting that the PCs are orthogonal to each other while their proportion represents that the variances decrease gradually. Above all, PCA was used for reducing the complex data sets and extracting the key variables that account for maximal variances in the multidimensional data sets. Independent-sample t-test was used to select the most significantly different PCs $(p<0.05)$ to effectively discriminate diabetes group and health group and these PCs were finally loaded to LDA to generate diagnostic algorithms. These steps are all carried out in SPSS software package (SPSS Inc. Chicago).

\section{Results and Discussion}

Figure 2 shows the normalized mean Raman spectra obtained from 39 diabetic blood Hb samples and 37 healthy blood $\mathrm{Hb}$ samples, with the standard deviations overlying as shaded color fill (shown on the top). There is a similar spectra profile between these two groups. Prominent Raman peaks at 568, 620, $676,753,793,898,970,1002,1126,1172,1228$, $1341,1372,1450,1562,1580,1602,1621$ and $1660 \mathrm{~cm}^{-1}$ were obviously observed in both diabetic and normal $\mathrm{Hb}$ samples, among which, the most intense Raman bands were found to locate at 1002, 1562, 1580 and $1621 \mathrm{~cm}^{-1}$. To highlight the subtle differences between diabetic and normal $\mathrm{Hb}$ samples more visually, the difference spectrum is shown (bottom in Fig. 2). Obviously, the differences are mainly reflected in the variation of relative peak intensity, which indicates that major differences may exist in the content of certain compositions. The vertical dotted line in the plot indicates the position where major differences exist. The band at $1228 \mathrm{~cm}^{-1}$, which is assigned to the $\mathrm{C}-\mathrm{H}$ methine in-plane bending vibration and glutathione, ${ }^{9,11}$ loses intensity as $\mathrm{Hb}$ stayed long time in high blood glucose level, whilst the band at 1562, 1580 and $1621 \mathrm{~cm}^{-1}$ appears to gain intensity. Among them, $1562 \mathrm{~cm}^{-1}$ is attributed to $\mathrm{C}_{\beta} \mathrm{C}_{\beta}$ stretching vibration, $1580 \mathrm{~cm}^{-1}$ is attributed to phenylalanine

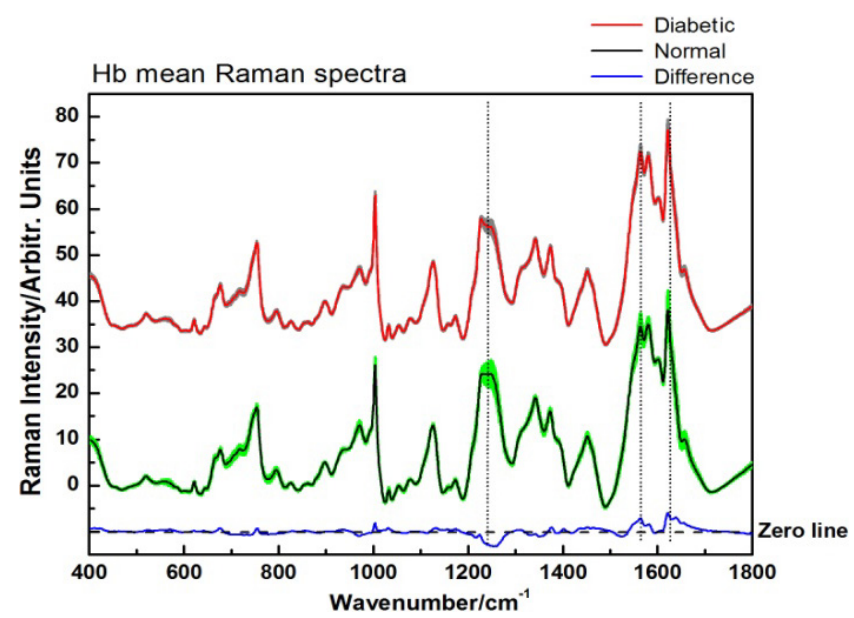

Fig. 2. (Color online) The normalized mean Raman spectra obtained from 39 diabetic blood Hb samples (red line) and 37 healthy blood $\mathrm{Hb}$ samples (black line), with the standard deviations overlying as shaded color fill. Also shown at the bottom is the difference spectrum. The vertical dotted line in the plot reflects the main difference. 


\section{J. Lin et al.}

and $\mathrm{C}_{\alpha} \mathrm{C}_{m}$ asymmetrical stretching mode ${ }^{9}$ which also have contribution to $1621 \mathrm{~cm}^{-1}$. These highfrequency peaks have been reliably related to vinyl group planarity with respect to the porphyrin core, by Smulevich et $a l .{ }^{12}$ So, the high blood glucose level not only has influenced on protein function, but also affects the heme's structure. More detailed assignments of all peaks are shown in Table 1.

In order to test the capability of blood $\mathrm{Hb}$ Raman spectra for differentiating diabetes from normal, PCA combined with LDA was performed on the pre-processed Raman data. After that, Independent-sample $t$-test on the obtained $\mathrm{PCs}$ showed that three PCs (PC1, PC2 and PC3) revealed the most significant diagnostic features $(p<0.05)$ for discriminating diabetic group and normal group. Finally, to incorporate all significant Raman spectral features, LDA was used to generate diagnostic algorithms using the PC scores for the three most significant PCs (PC1, PC2 and PC3).

Figure 3 shows a three-dimensional (3D) scatter plot of the three PC scores (PC1, PC2 and PC3) between the diabetic and normal groups. These two groups demonstrate two obvious separated regions though there still exist a little overlap. The sensitivity and specificity of this method for identifying

Table 1. Raman peak positions and tentative assignments. ${ }^{8-10}$

\begin{tabular}{|c|c|}
\hline $\begin{array}{l}\text { Raman } \\
\text { shift }\left(\mathrm{cm}^{-1}\right)\end{array}$ & Assignments \\
\hline 568 & $\nu\left(\mathrm{Fe}-\mathrm{O}_{2}\right)$ \\
\hline 620 & Phenylalanine \\
\hline 676 & $\delta(\text { pyr deform })_{\text {sym }}$ \\
\hline 753 & $\nu($ pyr breathing $)$, tryptophan, \\
\hline 793 & $\nu$ (pyr breathing) \\
\hline 898 & $\mathrm{p}: \mathrm{C}-\mathrm{C}$ skeletal, $\delta(\mathrm{C}-\mathrm{O}-\mathrm{H})$, glutathione, \\
\hline 970 & p: Skeletal vibration, glutathione \\
\hline 1002 & Phenylalanine \\
\hline 1126 & $\nu\left(\mathrm{C}_{\beta}\right.$-methyl $), \mathrm{L}$-Valine \\
\hline 1172 & $\nu(\text { pyr half-ring })_{\text {asym }}$, Tyrosine \\
\hline 1228 & $\delta\left(\mathrm{C}_{\mathrm{m}} \mathrm{H}\right)$, glutathione \\
\hline 1341 & $\nu(\text { pyr half-ring })_{\mathrm{sym}}$ \\
\hline 1372 & $\nu(\text { pyr half-ring })_{\text {sym }}$, glutathione \\
\hline 1450 & $\delta\left(\mathrm{CH}_{2} / \mathrm{CH}_{3}\right), \mathrm{D}-(\mathrm{C})$-galactosamine, \\
\hline 1562 & $\nu\left(\mathrm{C}_{\beta} \mathrm{C}_{\beta}\right)$ \\
\hline 1580 & $\nu\left(\mathrm{C}_{\alpha} \mathrm{C}_{\mathrm{m}}\right)_{\text {asym }}$, Phenylalanine \\
\hline 1602 & $\nu(\mathrm{C}=\mathrm{C})_{\text {vinyl }}, \nu\left(\mathrm{C}_{\mathrm{a}}=\mathrm{C}_{\mathrm{b}}\right)$ \\
\hline 1621 & $\nu(\mathrm{C}=\mathrm{C})_{\text {vinyl }}, \nu\left(\mathrm{C}_{\mathrm{a}}=\mathrm{C}_{\mathrm{b}}\right)$ Phenylalanine \\
\hline 1660 & Amide $\mathrm{I}, \nu(\mathrm{C}=\mathrm{O})+\nu(\mathrm{C}-\mathrm{N})+\delta\left(\mathrm{NH}_{2}\right)$ \\
\hline
\end{tabular}

Notes: $\nu$ and $\delta$ : In-plane modes, sym: symmetric, asym: asymmetric, p: protein.

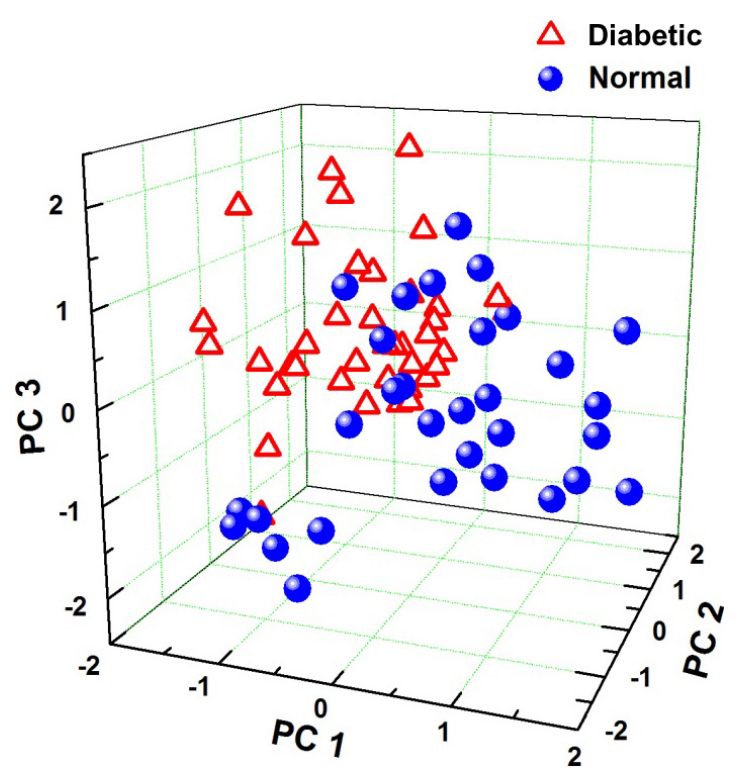

Fig. 3. (Color online) A 3D mapping of the PCA scores for the diabetic group (red triangle) and the normal group (blue circle) (PC1, PC2 and PC3 were chosen as the $x-y-z$ axis).

diabetes by the blood Hb Raman analysis were calculated to be $92.3 \%$ and $73.0 \%$, respectively instead of $84.6 \%$ and $70.3 \%$ for PC1 vs PC2 and $79.5 \%$ and $70.3 \%$ for PC1 vs PC3. To confirm the effectiveness of the diagnostic algorithm based on PCA-LDA technique for blood $\mathrm{Hb}$ differentiation, the receive operator characteristic (ROC) curve,

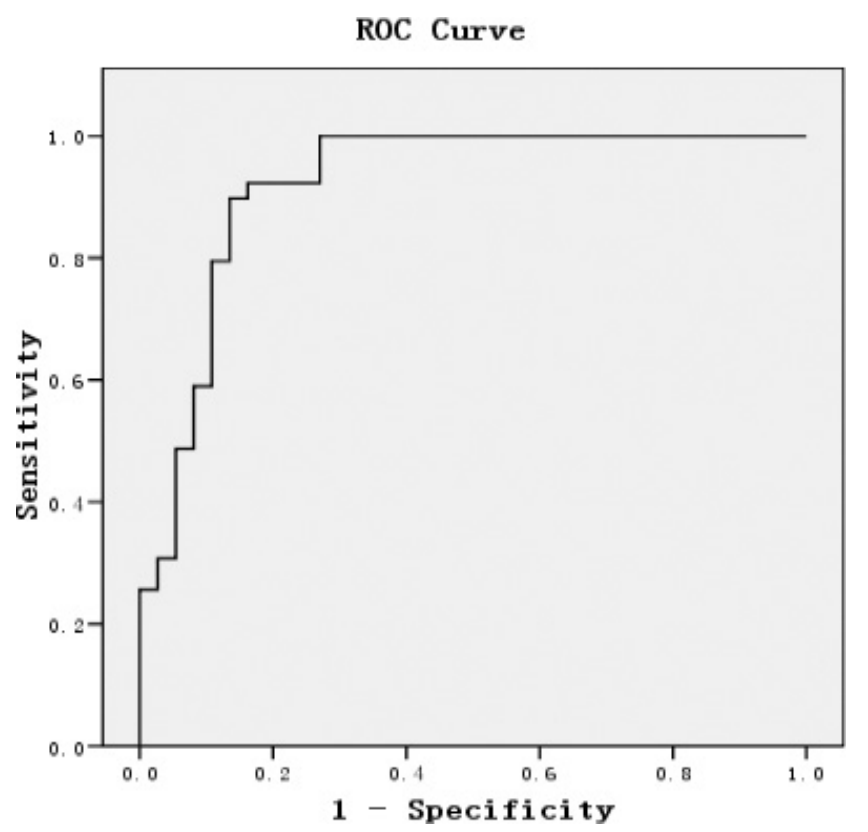

Fig. 4. ROC curve of the discrimination result using the PCALDA-based spectral classification. The integrated area under the ROC curve is 0.920 . 
which is a comparison of two operating characteristics (true and false positive rates) as the threshold level changes, was generated and the result was shown in Fig. 4. The area under the ROC curve was 0.920 , showing a relatively high accuracy of differentiation with Raman spectra of blood $\mathrm{Hb}$.

\section{Conclusion}

To the best of our knowledge, this is the first time that RS was employed to analyze human $\mathrm{Hb}$ samples for the differentiation between normal volunteers and diabetic patients. The tentative assignments of the measured Raman bands of $\mathrm{Hb}$ were performed, indicating that the high blood glucose level not only has influence on protein function, but also on the heme structure. RS combined with multivariate analysis (PCA-LDA) was able to distinguish the blood $\mathrm{Hb}$ of diabetic patients from that of healthy subjects with a diagnostic sensitivity and specificity of $92.3 \%$ and $73 \%$, respectively. Anyway, our preliminary study demonstrates RS that provides a new and noninvasive method for differentiation of normal volunteers and diabetes patients based on $\mathrm{Hb}$ Raman spectra. Our future work will focus on combing surface-enhanced Raman spectroscopy (SERS) method with multivariate analysis method to improve the accuracy for the diagnosis of diabetes.

\section{Acknowledgments}

This work was supported by the National Natural Science Foundation of China (Nos. 11274065, 61210016, 61308113, 61178090 and 81101110), the Science and Technology Project of Fujian Province (No. 2012J01254 and 2013J01225) and Program for Changjiang Scholars and Innovative Research Team in University (No. IRT1115).

\section{References}

1. K. F. Hanssen, H. J. Bangstad, O. BrinchmannHansen, K. Dahl-Jørgensen, "Blood glucose control and diabetic microvascular complications: Longterm effects of near-normoglycaemia," Diabet. Med. 9(8), 697-705 (1992).

2. C. S. Fox, S. Coady, P. D. Sorlie, S. Dagostino-RB, M. J. Pencina, R. S. Vasan, J. B. Meigs, D. Levy, P.
J. Savage, "Increasing cardiovascular disease burden due to diabetes mellitus: The Framingham heart study," Circulation 115(12), 1544-1550 (2007).

3. E. J. Gallagher, D. L. Rolth, Z. Bloomgarden, "Review of hemoglobin $\mathrm{A}_{1 c}$ in the management of diabetes," J. Diabetes 1(1), 9-17 (2009).

4. S. Y. Feng, R. Chen, J. Q. Lin, J. J. Pan, G. N. Chen, Y. Z. Li, M. Cheng, Z. F. Huang, J. S. Chen, H. S. Zeng, "Nasopharyngeal cancer detection based on blood plasma surface-enhanced Raman spectroscopy and multivariate analysis," Biosens. Bioelectron. 25(11), 2414-2419 (2010).

5. H. W. Han, X. L. Yan, R. X. Dong, G. Ban, K. Li, "Analysis of serum from type II diabetes mellitus and diabetic complication using surface-enhanced Raman spectra (SERS)," Appl. Phys. B 94(4), 667$672(2009)$.

6. I. Barman, N. C. Dingari, J. W. Kang, G. Horowitz, R. R. Dasari, M. S. Feld, "Raman spectroscopybased sensitive and specific detection of glycated hemoglobin," Anal. Chem. 84(5), 2474-2482 (2012).

7. J. Zhao, H. Lui, D. I. Mclean, H. S. Zeng, "Automated autofluorescence background subtraction algorithm for biomedical Raman spectroscopy," Appl. Spectrosc. 61(11), 1225-1232 (2007).

8. J. Q. Lin, R. Chen, S. Y. Feng, J. J. Pan, Y. Z. Li, G. N. Chen, M. Cheng, Z. F. Huang, Y. Yu, H. S. Zeng, "A novel blood plasma analysis technique combining membrane electrophoresis with silver nanoparticle based SERS spectroscopy for potential applications in non-invasive cancer detection," Nanomed Nanotechnol. Biol. Med. 7(5), 655-663 (2011).

9. B. R. Wood, P. Caspers, G. J. Puppels, S. Pandiancherri, D. McNaughton, "Resonance Raman spectroscopy of red blood cells using near-infrared laser excitation," Anal. Bioanal. Chem. 387(5), 1691-1703 (2007).

10. W. R. Premasiri, J. C. Lee, L. D. Ziegler, "Surfaceenhanced Raman scattering of whole human blood, blood plasma, and red blood cells: Cellular processes and bioanalytical sensing," J. Phys. Chem. B 116 (31), 9376-9386 (2012).

11. J. D. Gelder, K. D. Gussem, P. Vandenabeele, L. Moens, "Reference database of Raman spectra of biological molecules," J. Raman Spectrosc. 38(9), 1133-1147 (2007).

12. G. Smulevich, S. Z. Hu, K. R. Rodgers, D. B. Goodin, K. M. Smith, T. G. Spiro, "Heme-protein interactions in cytochrome c peroxidase revealed by site-directed mutagenesis and resonance Raman spectra of isotopically labeled hemes," Biospectroscopy 2(6), 365-376 (1996). 